\title{
Conflictos de la identidad sexual en la infancia
}

\section{RESUMEN}

Se ilustran con 5 viñetas clínicas de niños en posición homosexual dos teorías psicogénicas de la homosexualidad, la de J.Lacan para la homosexualidad masculina y la de H.Deutsch para la homosexualidad femenina.

Se revisa la bibliografía sobre la etiopatogenia de las identificaciones homosexuales y de las alteraciones de la identidad de género.

Palabras clave: Homosexualidad. Infancia. Trastorno de identidad de género.

\section{Introducción}

El interés de este trabajo, nace de la observación de niños y adolescentes consultantes en un Centro de Salud Mental Infanto-Juvenil (CSMIJ), en los que destaca una identidad homosexual o conflictos con su identidad sexual, a pesar de que los motivos de consulta sean otros: trastornos de comportamiento, de relación con los compañeros, fracaso escolar. No se trata tanto de alteraciones de la identidad sexual ligadas a patologías graves, como en el caso de las psicosis, sino más bien a las dificultades que surgen en el niño $\mathrm{y}$ adolescente, debidas a dichas identifica-

\section{SUMMARY}

We explain with five clinics vignettes on childhood homosexuality position, two psychogenetic theories of the homosexuality: The theories of J.Lacan on male homosexuality and the theories of H.Deutsch on female homosexuality.

We revise the etiogenetic factors in the ego-syntonic homosexuality and the gender identity disorder, in actual bibliography.

Key words: Homosexuality. Childhood. Gender identity disorder.

ciones homosexuales, más o menos en proceso de fijación o como defensa frente a ciertos conflictos evolutivos, a veces también importantes, como en el caso de los trastornos de personalidad infantil. Unos y otros casos habrán de ayudarnos a conocer mejor la dinámica de las identificaciones homosexuales en el período infanto-juvenil.

\section{Justificación}

El interés en abordar el tema de las identificaciones, comportamientos y dinámica de la homosexualidad y los conflictos con la identidad de género durante la 
infancia-adolescencia, es para que lo tengamos presente en nuestro trabajo clínico cotidiano, al menos por dos razones que desarrollamos a continuación: por el origen infantil de la homosexualidad y de otras variantes de la sexualidad, más o menos conflictivas para el sujeto, y porque las dificultades con la identidad de género tiende a asociarse con una mayor vulnerabilidad para el trastorno mental.

\section{Origen infantil de la orientación homo- sexual.}

Varios trabajos hacen alusión a la correlación existente entre el comportamiento homosexual del adulto y sus manifestaciones en la infancia. Una disconformidad latente respecto del género durante la infancia, correlaciona con una mayor expresión de la homosexualidad en el adulto. De los sujetos que demostraban homosexualidad latente en el estudio de Australia (2), resultaron homosexuales: el $12-15 \%$ de los varones y el 5-10\% de las mujeres. El comportamiento infantil en relación al género (5), relatado por el propio sujeto o por su madre, parece correlacionar con la homosexualidad posterior.

Un estudio (3) transcultural, muestra diferencias en las conductas infanto-juveniles entre las mujeres que luego se definen como homo u heterosexuales. Estas diferencias deberemos tenerlas en cuenta para el mejor reconocimiento de la conducta homosexual femenina, así como para evitar algunos riesgos. Las lesbianas fueron sexualmente más activas de niñas y se interesaron antes por chicas que las heterosexuales por chicos. Sin embargo, de mayores, se interesaron más tarde por mujeres que las heterosexuales por hombres. La atracción de las lesbianas por hombres, fue más temprana que la de las heterosexuales por mujeres. La edad del primer contacto sexual fue más temprano en lesbianas, teniendo como compañero sexual en proporción semejante al hombre y a la mujer (existiendo riesgo de embarazo adolescente en lesbianas), mientras que para la heterosexual, el primer contacto sexual era más frecuente con hombres. Las lesbianas se definen como homosexuales más tarde que las heterosexuales como tales. Concluyen los autores que las normas de comportamiento heterosexual impactan en el desarrollo de la homosexualidad femenina y de la identidad, pero no la obliteran.

Phillips (6) encontró que lo que diferenciaba a las mujeres homosexuales de las heterosexuales, más que el comportamiento, eran algunas vivencias que se atribuían a si mismas desde la infancia: imaginarse con un carácter masculino, desear llegar a ser madre, preferencia por los juegos de niños y considerar a un marimacho semejante a un niño.

Los varones homosexuales (4) se diferenciaron de los heterosexuales, por los comportamientos infantiles tipificados para cada sexo; aunque entre los homosexuales hubo una mayor varianza de comportamientos que entre los heterosexuales.

Vulnerabilidad para trastorno mental de la persona homosexual.

Algunos estudios destacan que la homosexualidad es un factor de riesgo para la salud mental. Las lesbianas (7) se muestran más proclives a padecer depresiones, 
abuso de sustancias e intentos de suicidio que la población general; parece que posiblemente sea el rechazo social junto a la presencia de otros factores de riesgo, quienes facilitan esta propensión en ellas. El afán familiar (8) por corregir expresiones femeninas durante la infancia, llevan a que el homosexual masculino adulto tenga dificultades de expresar ciertos afectos. El niño con manifestaciones homosexuales, es más vulnerable al trastorno mental, porque durante la infancia, el rechazo ejercido por sus compañeros y adultos, respecto a comportamientos propios del género contrario, son muy fuertes. Este riesgo para la salud mental, hace que los casos nos lleguen a la consulta por diversos motivos, a veces únicamente reactivos a la situación. En el trabajo clínico no debemos minusvalorar esta cuestión, con frecuencia no dicha, sea con el paciente, su familia o el entorno escolar, sobre todo en el caso de los adolescentes con una clara identidad homosexual.

\section{Objetivos}

Este trabajo quiere desarrollar un doble objetivo:

1.- Explorar a través de casos clínicos, en la infancia y adolescencia, las teorías psicogenéticas sobre la homosexualidad.

2.- Previamente realizaré una revisión bibliográfica sobre la epidemiología y factores etio(pato)génicos de los conflictos de identidad de género, para terminar examinando las teorías psicoanalíticas que explican la formación de la homosexualidad durante la infancia.
Epidemiología de los trastornos de identidad de género.

Algunos autores como Becker o Fernández Rivas(33), estiman que el conflicto de identidad sexual, entendido como contradicción entre el propio sexo biológico y la identidad de género (tipo transexualismo o travestismo), presenta una prevalencia baja en la infancia y adolescencia, aunque está poco estudiada. En el adulto, parece corroborarse una mayor prevalencia de la transexualidad en varones, del orden de $2: 1$ a 8:1, calculándose prevalencias de 1-3 casos cada 10.000 hombres, frente a 1-3/100.000 mujeres. Zucker (1) recoge información desde 1978 a 1995, señalando que los trastornos de la identidad de género se dan en mayor proporción en niños que en niñas, en una relación de 6.6:1. Una hipótesis para explicar esta diferente prevalencia, es que a las niñas se les acepta más fácilmente el mostrar comportamientos de ambos géneros.

Además, en el período infanto-juvenil, más allá del rechazo del sexo biológico, tipo transexual, se nos plantea si el conflicto entre la identidad sexual (sexo biológico) y de género (modo de ser ligado al sexo), es permanente o pasajero, egosintónico o egodistónico, lo que nos conduce al tema de la formación de la identidad ligada al sexo, destacando de entre todas las posibles orientaciones, la homosexual.

Durante la infancia y la adolescencia se va a desarrollar una de las líneas troncales de evolución de la persona, esto es, el eje psicosexual, con todas sus posibles variaciones, según que la fuente pulsional sea genital o pregenital(oral, anal, voyeur, frotteur, etc); según que el objeto 
sexual sea no humano (zoofilia..) o humano, bien homo o heterosexual (o que una propiedad particular del objeto, como la edad, sea clave en la elección: paidofilia, gerontofilia, etc.), o que el fin pulsional esté más o menos ligado a la agresividad (violación, sadismo, masoquismo..); es decir, que la sexualidad humana puede tomar muchas y complejas expresiones.

Por tanto, como en la infancia es cuando se está empezando a jugar este desarrollo complejo de la sexualidad, nuestro interés es el de analizar la psicodinamia de la orientación homosexual, la más frecuente después de la heterosexual. En nuestros pacientes, estas identificaciones homosexuales, se muestran casi siempre egosintónicas, aunque participen de conflictos relacionales, estas identificaciones pueden estar fijadas o formar parte de una defensa frente a otros conflictos, a veces internos y con más frecuencia relacionales.

Factores etio(pato)génicos de la homosexualidad y de las alteraciones de la identidad de género en la infancia.

Factores ambientales:

Diferentes estudios señalan que existe una asociación entre factores ambientales y variaciones de la identidad sexual. Se investigó si ciertos factores favorecen la aparición de la homosexualidad, por ejemplo, si la presencia de homosexuales en la familia, aumentaba su prevalencia, o si los clásicos factores de riesgo de la organización familiar, como el divorcio, la enfermedad mental de los padres, los conflictos relacionales graves, las relaciones incestuosas o el que los niños sean adoptados, tienen influencia respecto del cambio en la identidad sexual esperada como heterosexual. Veremos al analizar estos diversos factores, que los conflictos relacionales, en general, pueden trasladarse a conflictos en la identidad de género.

Presencia de homosexuales en la familia:

De los estudios revisados por P.Cameron (12) se deduce que los hijos de homosexuales, según ellos mismos, tienden a tener más dificultades familiares atribuibles a que el padre sea homosexual, así como que, en mayor proporción que la población general, se reconocen como homo o bisexuales. Sin embargo, el descubrimiento de la homosexualidad de un hermano (13), no influye en la identidad sexual propia. Los hermanos que eran ambos homosexuales, presentaron similar disconformidad de género durante la infancia.

Conflictos familiares:

Reckers, (9) mediante el test que lleva su nombre, encontró que los trastornos de identidad de género, se dan con mayor frecuencia entre los hijos de padres con trastornos psiquiátricos o con conflictiva relacional. El mismo autor (10) encuentra que los hijos de padres separados presentan, con mayor frecuencia que la población general, dificultades respecto al género. Zucker (11) y otros discuten si el orden de nacimiento de los hermanos es un factor importante cara al trastorno de identidad de género.

Sexualidad intrafamiliar:

El estudio de Cameron (14) sobre más de 5000 personas, demuestra que en los 
grupos homo y bisexuales, hay una mayor proporción de relatos de incesto, que entre los heterosexuales. La sobreestimulación erótica en la infancia (15), puede favorecer la homosexualidad del varón.

Adopción, apego y sexualidad:

Blumenthal (16) describe el caso de una niña adoptada que rechaza su identidad sexual femenina, debido a dificultades en sus relaciones de apego y elaboración de pérdidas. Gilmore (17) observa también el caso de una niña adoptada y los efectos que el traumatismo de la separación tiene sobre la identidad sexual. La interacción entre apego, dificultades de separación e identificación homosexual, es uno de los caminos que exploramos en nuestro estudio.

Factores biológicos:

Se exploran factores biológicos, a la búsqueda de razones que expliquen variaciones respecto de la identidad de género; ahora se trata de una perspectiva no ambiental sino temperamental.

Genéticos:

El hecho de que nos interesemos por los mecanismos psicológicos de configuración de la homosexualidad, no obsta para reconocer la presencia de un factor constitucional hereditario, pues como demostró F.J. Kallmann, estudiando a 40 gemelos homocigóticos, en 37 de ellos ambos eran homosexuales, mientras que en 23 dicigóticos, sólo 3 de las parejas de hermanos eran homosexuales. En gemelos (24), parece que la orientación sexual se hereda más que otros modos de ser, pues se observa que la concordancia respecto a la disconformidad con el género en la infancia, es mayor que la de otros rasgos ha- bitualmente transmitidos por la familia. Se buscan apoyaturas en la transmisión genética, pero los estudios (25) sobre la relación entre algunas anomalías del cromosoma $\mathrm{X}$ y la homosexualidad, no son concluyentes. Un curioso estudio de Zucker (26), encuentra que significativamente, los niños con trastorno de la identidad de género, son con más frecuencia zurdos, lo que le hace pensar en la influencia de factores neurobiológicos. Este mismo autor y otros (27) demostraron que los niños de 8 años con trastornos de la identidad de género, eran juzgados por fotógrafos, sin conocimiento previo de la cuestión que se analizaba, como más atractivos que los del grupo control. Lo que les hace pensar en un factor constitucional. Este juicio lo hacían apreciando el torso y la cara.

\section{Hormonales:}

Se postula (28) que un descenso de testosterona en períodos críticos del desarrollo, como es el postnatal, prolonga la hiperprolactinemia, afectando a núcleos hipotalámicos, que influyen sobre el comportamiento psicosexual.

\section{Psicodinamia del desarrollo sexual.}

Hemos visto que algunos factores ambientales se asocian a la presencia de conflictos en la identidad sexual. Ahora veremos los mecanismos explicativos de cómo esos factores familiares, a través de la interacción entre el psiquismo familiar y el individual, permiten comprender los cambios en el proceso de identificación sexual.

Diferentes artículos de orientación psicoanalítica, nos muestran los mecanis- 
mos implicados en los trastornos o conflictos de la identidad de género en niños, encontrando que ciertas dificultades relacionales están incidiendo en la identificación sexual. En ocasiones, la identificación sexual desviada es una defensa frente a un conflicto, mientras que, otras veces, esa identificación está más fijada y el mecanismo subyacente sirve para explicar el cambio de identidad de género. Parece que es en el período pre-edípico donde se juega el origen de los trastornos de identidad de género.

Sobre la precocidad de las identificaciones sexuales:

Ajuriaguerra (29) recopila varios trabajos, concluyendo que basta preguntar al homosexual adulto, para saber que éste se reconoce precozmente. Sin embargo, se trata de un proceso, no de algo fijo desde el principio, por eso S.Freud dice que, «hasta pasada la pubertad no tomará de forma definitiva el comportamiento sexual».

G.W.Henry señala que 4 de cada 5 homosexuales, dicen que sus primeras manifestaciones son anteriores a los 9 años; y 1 de cada 5, reconocen su comienzo en la pubertad. Quedando establecida a los 1725 años.

La homosexualidad femenina parece ser reconocida más tarde, en un $61 \%$ de las encuestadas, contestan que a los 14 años, como media (según F.E.Kenyon).

Loeb (18) estudia algunos niños que ya en edad temprana muestran síntomas transexuales, que en algún caso tenía que ver con una identificación con su abuela agresiva, seductora y castradora. El mismo autor (19) narra el caso de un niño de 5 años con deseo de cambio de género, li- gado a un conflicto de apego simbiótico con la madre, que a través de la transferencia se resuelve cuando el niño logra individuarse y aceptar su identidad masculina. Meyer (20) nos muestra que en los casos por él observados, los trastornos de la identidad de género se dan en el período de separación-individuación, asociándose a conflictos importantes o traumas sufridos en este período. McDevitt (21) estudia los componentes pre-edípicos de los trastornos de identidad sexual. A partir de un caso se da cuenta que el inconformismo con la propia identidad sexual, puede ser una formación de compromiso respecto de un conflicto intrapsíquico.

\section{Sobre la identificación con la madre y} la dinámica edípica

La reflexión sobre algunos casos nos proporciona un conocimiento de los mecanismos que configuran el origen de la homosexualidad en el niño, que luego se fijará definitivamente en el adulto.

Chused (22) nos muestra a través de tres análisis de homosexuales, cómo algunos juegos que éstos realizaban durante la adolescencia, frente al espejo, tienen que ver con una identificación con la madre, sea madre perdida, madre enfadada, o madre que se desea poseer y dominar. Haber (23) describe el caso de un niño de 3 años, que mostró deseo de ser niña. Al parecer se relacionó con una dificultad de identificarse con figuras masculinas (padre emocionalmente ausente, muerte y accidente del abuelo y del tío). El niño presentó tempranamente un miedo a la pérdida de objeto. Además la madre deseaba, una hermana durante su infancia, y una hija 
de adulta; también jugó un papel importante la fantasía compartida entre madre e hijo, sobre la belleza y poder de las mujeres (madre y abuela).

L.Bender, estudió niños de 4 a 12 años, distinguiendo tres grupos:

- Aquellos que hacen una identificación homosexual, porque viven como inadecuada la otra imago parental.

- Identificación con el progenitor del sexo contrario, por ser odiado o temido el del propio sexo.

- Aquellos que se crían sin padres y tienen dificultades para identificarse con una figura de importancia para él.

S.Lebovici (30) y L.Kreisler, creen que la identificación materna y la pasividad, son señales de alerta que anteceden a la homosexualidad fijada.

Stoller (30), que estudia el travestismo infantil a la edad de 2 años, describe como manifestaciones precoces, el afeminamiento, la desvalorización del pene y la belleza del niño, junto a una madre que permite la separación de la persona pero no de su feminidad, mediante un exceso de contacto o promiscuidad cutánea, de forma que el niño con su cuerpo parece llenar la frialdad narcisista de la madre. Describe una madre insatisfecha en general y sexualmente del marido, aunque tenga orgasmos, de forma que el padre cuenta poco en la unión/separación madre-hijo. Stoller (31) estudia otros comportamientos homosexuales, como el travestismo, subrayando el comienzo precoz, a los 1-2 años, en gestos y elección de ropas. Rehuyen la actividad y agresividad de los varones, prefiriendo la dramatización, lectura, conversación...Saben que tienen pene, pero les gustaría ser chicas. La precocidad del afeminamiento, la desvalorización del pene y la belleza del niño, son para Stoller señales precoces.

Teorías sobre la génesis de la homosexualidad:

Ana Freud nos proporciona unos factores que componen una teoría de la homosexualidad:

- Tendencias de la constitución.

- Mantenimiento de esquema narcisista primario.

- Erotización de fase anal pasiva.

- Fijación anaclítica (el sexo es secundario a la dependencia personal).

- Envidia del pene.

- Sobreestimación del pene en fase fálica (impide al chico aceptar un objeto castrado).

- Complejo edípico negativo.

Mientras que empuja a la heterosexualidad:

- Complejo edípico positivo y fálico.

- Miedo a la castración (en contra del Edipo negativo).

- Asco frente a fase anal.

Hay otros factores que pueden inducir a la homosexualidad, como un hipererotismo infantil mal encauzado, por ejemplo debido a la seducción de un pederasta. Temores fantasmáticos de los padres respecto a sus propias tendencias homosexuales. Búsqueda de afecto, en niños carenciados, etc..

Este conjunto de factores y mecanismos parecen estar bien sintetizados por J.Lacan. Veamos la explicación de J.Lacan (32) sobre el triple mecanismo de la homosexualidad masculina. 
1.- «Una fijación afectiva a la madre, fijación en relación con la cual es fácil comprender que determine la exclusión de toda otra mujer».

2.- «La ambivalencia narcisista con la cual el sujeto se identifica al objeto de amor con su propia imagen especular».

3.- «En el trasfondo del psiquismo, la intervención realmente castradora a través de la cual la madre ha canalizado su propia reivindicación viril».

La homosexualidad femenina fue estudiada por H.Deutsch, señalando los siguientes mecanismos:

1.- En la prepubertad, la niña es atraída por otra niña doble de su yo (elección narcisista).

2.- Identificación con el agresor, en casos en que lo era su padre.

3.- Nostalgia de la madre (homosexualidad que reproduce una relación materno-filial).

\section{Material y Método.}

Se examinan cinco casos clínicos que nos servirán para confrontar las teorías psicogénicas de las conductas homosexuales masculina y femenina en niños y adolescentes. El estudio de casos únicos es el que consideramos adecuado para comprender los mecanismos que intervienen en el proceso de organización sexual.

Las viñetas de los casos clínicos se muestran en el apartado siguiente, que se deben consultar según se van citando en el texto de la discusión con las letras (a,b,c,d,e).
Resultados y discusión.

En la relación vincular inconsciente de la madre con el niño con identificación homosexual, se observa una madre para quien el hijo es todo suyo(caso a) o un hijo para quien la madre lo es todo (o lo único que tiene, o su agresora)(caso b); al mismo tiempo aparece un padre amenazante, temido y castrante o un padre descalificado, menospreciado o castrado. En el mismo niño suelen estar presentes estas dobles imagos, si bien suele predominar alguna de ellas(en lo real o en lo imaginario). Esta vivencia de peligro que amenaza su narcisismo, le empuja a elegir su imagen propia como objeto de amor (b). (punto 2 de Lacan).

Para la madre fálica, el niño es suyo, a veces el niño siente que para existir, vivir o desear, ha de ser el falo o deseo de la madre (b) (punto 3 de Lacan).

Por eso, en algún caso la angustia de castración está todavía demasiado ligada a la de separación (caso c), mostrando en su evolución homosexual, huellas de aquel proceso (angustia de separación, fijación a objetos transicionales que se transforman en fetiches, ligados a un deseo sexual parcial como fijaciones anales...). La identificación homosexual es una forma de crear un puente que salta sobre la angustia de separación. (punto 1 de Lacan).

Para lograrse la desvinculación fálica, la madre debe permitir al hijo pensar que el padre puede poseer el falo, así como pensar que ella misma no lo posee, incluso con el dolor de saber que, aceptar esta castración, además conlleva la pérdida-separación del hijo. La aceptación de la falta simbólica, mediada por la castración sim- 
bólica, pone fin o punto y aparte, al proceso de separación de la unión dual, que marca y registra el espacio simbólico de la separación (para C.Le Guen, es con la aparición del extraño cuando se inicia este proceso, que se cerrará con la presencia castradora o limitadora, cortante, decisiva, del padre (recordar a J.Oury en déscision) (punto 1 de Lacan).

Si la madre no da ese permiso superyoico, la madre favorece que el superyó arcaico se proyecte sobre un padre temido-castrador-vengativo, del que se huye por temor a una desmesurada castración; pueden darse varios mecanismos que favorecen la identificación del niño con la madre: 'tu eres mío' o ‘yo soy tuyo' como modo de evitar el sufrimiento de una pérdida (por ejemplo debido a una muerte inoportuna de la madre), puede convertirse también en 'yo soy como tú', una mujer, como principal identificación secundaria .(punto 2 de Lacan). Las dificultades en la separación pueden ser más graves, de forma que el niño puede encontrar unas defensas que preserven, en mayor o menor grado, su identidad: frente al 'yo es tú' psicótico, puede alcanzarse el 'tu y yo lo tenemos todo' de la unión dual, o que mediante el 'no puedo irme con otra', salve la identificación primaria a costa de modificar la identificación sexual.

La imago del padre puede estar deformada por la realidad práctica o por la realidad psíquica imaginaria, en parte debido a la relación madre-hijo, lo que da como resultante una identificación homosexual (tanto para superar una angustia psicotizante o de simbiosis, como para evitar la angustia de castración-aniquila- ción real, los pacientes con organizaciones límite se encuentran también en el centro de este torbellino, pero no logran poner fin a la inestabilidad identificatoria, de ahí que el polimorfismo sea mayor, y la integración del yo es mucho menor; la homosexualidad, en algunos casos, es una salida de los conflictos de la separación, que pueden proteger al sujeto de la inestabilidad propia de yo de las organizaciones límite, porque separa sexualidad de amor, integrando al yo y canalizando las pulsiones parciales hacia la genitalidad.

El padre no aparece ante el niño como la paradoja entre padre total y padre castrado en sentido simbólico, como portador de que la diferencia de sexos es inevitable, sino que se desmiente esta articulación, de forma que aparece como un padre-madre total, o un padre violento-vengativo, castrador por ser castrado (caso d).

La homosexualidad es una solución económica al dilema edípico precoz, pues concentra en la identidad sexual las crisis de integración del yo, tal como la despersonalización, extrañamiento...que surgen de la separación, al tiempo que evita las angustias de castración, ambas quedan suturadas con el cambio identificatorio, aunque a costa del desmentido de la castración.

En el paciente caso (a), que padece de parálisis histéricas, éstas se desencadenan cuando se encuentra reprimiendo su deseo homosexual y se enfrenta a la seducción de una chica que le propone relaciones heterosexuales. En este caso la histeria es una defensa contra el deseo heterosexual que encubre el homosexual; como en el caso Dora de S.Freud. 
La identificación sexual se produce a partir de los dos años, durante el proceso de separación y por tanto influido por éste. En ese período ya se consolidó la identificación primaria del yo-corporal, que proporciona unidad y continuidad al sentimiento de si mismo; junto al proceso de identificación sexual también se construye el de la diferenciación generacional. Como los procesos de identidad corporal y sexual son tan próximos (quizás la identificación sexual es la más precoz de las identificaciones secundarias), junto al de identidad intergeneracional, es por lo que los roles sexuales vienen tan ligados a lo gestual, al cuerpo, al estilo musical o melodía de la imagen inconsciente del cuerpo.

Para el caso de la homosexualidad femenina, el caso (e) nos ejemplifica las teorías de H.Deutsch, pues vemos la añoranza que la niña tiene de la madre, con la que reproduce, desdoblándose, la relación sexuada de ser niña para la mujer, así, establece la relación maternal de ser madre para la niña, no pudiendo identificarse como mujer sexuada y madre al mismo tiempo, dado que la imago de la madre no ha permitido desarrollar esa doble representación, que la niña busca por otros caminos, como es a través de la terapia (imagen maternante en la transferencia), o con su tía casada y con hijos, figura que solicita como sustitutiva de la madre, en la que conscientemente no reconoce ninguna fa1la. Este desdoblamiento de las identificaciones actuadas, también aparecen en sus dibujos. En este caso podemos observar la evolución de esta identificación homosexual, que no está fijada, que en parte se traslada a la transferencia conmigo (en una de las sesiones efectuó una introyección de lo que le interpretaba a partir de nombrar el contenido de los dibujos que realizaba, profusión de corazones más o menos rotos, introyección que gestualizaba mediante los brazos que recogen trozos de cosas que se hubieran derramado sobre la mesa, repetidamente, guardándoselo en si, y que en realidad fue una verdadera reedición de una identificación con un objeto de amor primario, cuando sobre este tema dibujaba; es posible que aunque provenga de una fijación anterior, este momento transferencial reedite la identificación con alguien de un sexo opuesto, lo que se acompañó más tarde de la expresión en el Centro donde reside, de su disconformidad con el sexo propio, de forma más o menos provocativa.

\section{Casos Clínicos}

- (Caso a). Una madre sin hijos, adopta a un sobrino cuando el niño tenía 1-2 años, debido a que su madre era toxicómana y negligente (recordemos las formas de contacto patológicas en las familias de toxicómanos). ¿Qué deseo hubo en la madre respecto del niño, de la tía adoptante y de su marido; qué fue del padre del niño?. La tía protege y acapara al niño frente a su hermana. En el discurso manifiesto parece haber una armonía parental adoptiva; el marido deja que su mujer tenga este hijo sin participar. El hijo muestra una angustia de pánico, ante cualquier proximidad del padre biológico, parece indicar la proyección de un superyó persecutorio por parte del niño sobre el padre real-biológico. Esta reacción del hijo parece consecuencia de una alianza entre madrastra e hijo: 
1.- La madrastra y el hijo se alían para defenderse del padre y dejarlo fuera de la unión madre-hijo, lo que además sirve para proteger al hijo (se trata del mito de Zeus y Rea su madre, frente al padre Crono; mito sobre la sucesión de generaciones y la angustia de devoración y castración).

2.- Esta alianza se repite en el caso c; El paciente ve una y otra vez la película de la sirenita; una sirena se enamora de un chico, pero su padre le prohíbe esa relación (debido a su identidad real de medio pez, es decir, que no es del todo humana); sin embargo, la madrastra (bruja) le promete que esa relación será posible (cambiar una cola por dos piernas), siempre que se alíe con ella frente al padre.

- (Caso b). Se trata de un paciente adolescente, de 15 años, que actualmente admite su condición de homosexual, y recuerda de su infancia escenas repetidas con su madre, tendida en el suelo o en la cama, borracha, teniendo que cuidarla (limpiarla y acostarla) con temor a que se muriera, al tiempo que recuerda que por aquella habitación pasaban a veces hombres.

Juan recuerda que le dejaban solo con su madre. Dice que nadie le quería. El padre, violento, estaba ausente, y la madre a veces le pegaba y le llamaba maricón; del mismo modo le insultaba su hermano, dos años mayor que él; mientras que con el hermano 3 años menor, se llevaba mejor.

Los padres se separan definitivamente cuando Juan tiene 11 años. A partir de los 6 años, recuerda ahora que se sentía mejor con las niñas, le gustaba ser como ellas, tranquilas, con sus juegos, prefiere hablar a realizar juegos de acción; a partir de los 8 años empeora en la escuela, teniendo que repetir dos cursos.

El motivo de consulta a los 14 años, es por una fuga del hogar y amenazas de suicidio, precisamente cuando tiene que ir a casa de su madre; con el compañero de la madre también se lleva mal (éste compañero de la madre ha estado ingresado en el Hospital por crisis paranoides a raíz de la transmisión mediática de un atentado terrorista).

El padre biológico, que también le ha tratado mal, nos llama en varias ocasiones para pedir hora, pero no consigue venir a la consulta, por no tener que oir que su hijo es homosexual y sentirse culpable de haber hecho algo mal.

Si bien Juan nunca destacó en los estudios, sí logra sublimar a través del teatro, de la cocina y la peluquería, que estudia y practica.

También se ha enamorado de chicos, pero le cuesta mucho descifrar el deseo de ellos (en este período se pinta el pelo, compra botas a lo Jane Jackson..); parece que exagerar el lenguaje extraverbal le ayuda a aclarar el metamensaje. Le gustaría ser como Winnie Huston o Jane Jackson. Últimamente está feliz, porque ha invitado al chico que le gusta a comer a casa.

Parece que desde pequeño estableció una fijación a la madre, como único punto de agarre cuando sintió que nadie le quería y que estaba solo. Se identifica a si mismo como objeto de amor, se cuidará a él como cuidó a su madre. La madre parece consolarse con el alcohol, se basta a si misma. El marido acaba separándose, des- 
pués de mucho tiempo de intentar limitarla. Su nuevo compañero, también es agresivo, paranoide, ambos padres son autoritarios pero incapaces junto a la madre de articular acuerdos, normas, reglas entre ellos.

- (Caso c). El paciente tiene 9 años, acude por ser muy movido, tener conductas extrañas, rechaza los juegos de niño por ser bruscos, se muestra seductor y amanerado; le gusta cambiarse de vestido y tener otro pelo, ser admirado. Tiene miedo a los cambios. Bruxismo nocturno. Se calma oliendo un trozo de bata o bailando (objeto transicional o identidad sexual gestual femenina).

Muestra dificultades, desde los dos años hasta la actualidad, en separarse de algunos objetos, como el chupete o los trozos de bata de su madre, que desde los 8 meses guarda y lleva siempre consigo, que ensucia y huele reiteradamente (placer anal-retentivo desplazado al olfato). Esta incorporación olfativa, parece sustituir a la incorporación oro-degustativa del comer-llenarse (pues no come y se adelgaza); disfruta chupando, pero no comiendo. Parece haber un clivaje entre la necesidad de comer (sentimiento de hambre y llenado) y el goce de oler y chupar. No puede incorporar, pero no puede poner freno al autoerotismo oro-olfativo. El fetiche o amuleto le sirve más para desmentir la separación que la castración. La masturbación olfatoria a veces se acompaña de noche de pequeñas emisiones de orina.

El trapo que la madre va recortando de su vieja bata conservada sin lavar en el armario, es un resto de objeto transicional que le da seguridad, le sirve de amuleto, y, como el fetiche, es un objeto desangustiante, seguro, que proporciona goce sexual parcial olfativo anal. Esta relación de objeto nace en el proceso de la separación (la angustia de separación dura un año en la guardería, comenzando a los dos años), es un objeto táctil, pero sobre todo olfatorio; el olfato es un sentido donde el dentro y fuera se confunden más fácilmente. El trapo retiene al objeto materno, conjurando parcialmente la angustia de separación, representa a las heces que se pierden como objeto interno o parte misma del cuerpo que se desprende, que se despeña, recuperando olfativamente las emisiones del cuerpo; el trapo es una esponja (de sudor, orina, suciedad del cuerpo y de lo que toca..). La erótica anal recupera al objeto que se pierde como resto no metaforizado (no puede metaforizar la separación, debido a esa fijación parcial; no puede tragar porque no metaforiza el tragar-llenarse de comida en tener plenitud; de forma que lo real y lo imaginario se solapan). Este goce anal retentivo es la fase previa a la fálico-uretral, de ahí los comportamientos obsesivoides que le defienden, junto con el fetiche, de la angustia de castración, que se evita. El fetiche desmiente la separación (reconoce que la hay pero se niega a percibirla, colocando el sistema simbólico en un nivel de transicionalidad). Por eso, incorporar la comida puede significar destruir al otro, devorarlo, poseerlo y en este proceso aniquilarlo; una fuerte pulsión oral incorporativa parece revelarse en que de bebé, si no le daban lo que quería o se lo quitaban, se golpeaba continuadamente la 
cabeza. En el único dibujo que espontáneamente hace la figura humana masculina, dibuja a drácula. Lo masculino se identifica con una agresividad oral, mientras que lo femenino con lo olfatorio anal-retentivo. La sirenita tiene rostro y pechos de mujer, pero cola de pez, cola que oculta el tener o no tener cola.

- (Caso d). Los dilemas sobre el padre castrador-castrado, aparecen en el caso Fernando, cuando el chico adolescente se levanta por las noches y se dirige a la habitación del padre, para tocarle los genitales. O como en el caso Manuel, cuyo padre les ataca porque descubre que ' $\mathrm{mi}$ madre y yo queremos castrarle'; el caso Fernando de algún modo piensa: 'yo soy el que por deseo de mi madre ejecutaré o he ejecutado (de ahí la necesidad de comprobación) la castración del padre'.

- (Caso e). Se trata de una niña de 10 años que acaba de ingresar en un Centro de protección del menor, debido a una negligencia crónica de parte de su madre. A pesar de la deficiencia en los cuidados maternos, la niña añora con una intensidad extraordinaria a su madre, de forma que en las visitas que quincenalmente le permiten, se pasan ambas llorando toda la entrevista, sin poder hablarse, más que de imprecisas promesas de volver a estar juntas en casa.

La niña acude a la consulta porque presenta conductas de rebelión y protesta, inadaptación al Centro, a la escuela, sordera que luego se comprobó que era un síntoma conversivo, llanto, dificultades para dormir; todo ello se enmarcaba en un sentimiento de negación de que su lugar de vida actual no era su casa. Posteriormente ha mostrado conductas de tipo sexual con las chicas mayores y de cuidado maternal con los niños pequeños. Además, a raíz de que le tienen que cortar el pelo por tener piojos, dice que prefiere tenerlo corto como un chico, porque ella quiere ser un chico. Sus aficiones son el baloncesto, la bicicleta y el fútbol, no le gustan las muñecas, aunque sí cuando era más pequeña. Tiene dificultades actuales para mantener cualquier juego que le requiera pararse y pensar, aunque se comunica bien a través de dibujos que comenta y le interesa que sean interpretados. Hasta el momento aparece una dificultad por separar lo bueno de lo malo, el ángel del demonio, quienes se transforman rápidamente entre si (como ocurre con sus conductas), se articulan con dificultad, como dos influencias contrarias. Su casa aparece cerrada, despoblada, habitada por sus objetos transicionales o de juego, como son la bicicleta, el balón y un objeto que condensa a tres, es una cesta de basquet, que al mismo tiempo es la calavera, el balón y el bebé; veremos como se despliega esa condensación a lo largo del tratamiento.

\section{Conclusiones.}

En primer lugar constatamos a través de nuestros casos clínicos, las hipótesis psicodinámicas de J.Lacan y H.Deutsch, sobre los mecanismos de formación de las identificaciones homosexuales masculina y femenina. Las identificaciones homosexuales pueden funcionar como mecanismos compensatorios de algunos conflic- 
tos que surgen en el proceso de separaciónindividuación.

Es de interés diferenciar las conductas homosexuales sin otras complicaciones psicopatológicas, en la infancia o adolescencia, de aquellas alteraciones de la identificación sexual dentro de un trastorno psiquiátrico. Si hoy en día no consideramos a la homosexualidad como un trastorno psiquiátrico en el adulto, y sabemos que este proceso psicosexual comienza en la infancia, será de la mayor importancia no pretender curarla o negarla, en definitiva, hacer de ella un conflicto que puede llevar al sujeto a un trastorno psiquiátrico, sino que conviene intentar que en el ámbito psicosocial cotidiano de desarrollo del niño o del adolescente, como son la familia o la escuela, pueda aceptarse al niño con conductas homosexuales tal como es, eso sí, descartando que esas conductas o identificaciones no formen parte de una defensa frente a otros conflictos, o sean parte de un trastorno psiquiátrico. Una vez que se descarta otro conflicto o trastorno psiquiátrico, que puede manifestarse a través de conductas más propias del otro género, será de interés para la salud mental del niño, seguirle a distancia, por si apare- cen otras señales de alarma o factores de riesgo de trastorno psiquiátrico, sin empecinarse en remodelar sus identificaciones de género, que por otra parte pueden modificarse espontáneamente a través de los cambios propios del proceso evolutivo.

Como hemos visto, ciertos factores de riesgo familiares hacen más conflictivas las identificaciones sexuales de los hijos, por eso hemos de atender a esos factores de riesgo, pero sobre todo, porque no sólo se asocian a una mayor presencia de variaciones en la identidad sexual, sino, y sobre todo, porque correlacionan con una mayor presencia de trastornos psiquiátricos, tal como se ha demostrado a partir de innumerables investigaciones.

Podría ser interesante, durante la adolescencia, habida cuenta de la soledad que constatamos en algunos chicos adolescentes homosexuales que nos consultan, que pudieran acudir a grupos de auto-apoyo e información homosexual, centros integrados en asociaciones reconocidas socialmente, con quienes intercambiar vivencias; o que hubiera grupos de padres de chicos homosexuales, por si quieren compartir sus experiencias. 


\section{BIBLIOGRAFIA}

1. Zucker KJ, Bradley SJ, Sanikhani M. Sex differences in referral rates of children with gender identity disorder:some hypotheses. J. Abnorm Child Psychol, 1997 Jun; 25(3):217-27

(2. Gangestad SW, Bailey JM, Martin NG. Taxometric analyses of sexual orientation and gender identity. J Pers Soc Psychol, 2000 Jun;78(6):1109-21

3. Whitam FL, Daskalos C, Sobolewski CG, Padilla P. The emergence of lesbian sexuality and identity cross-culturally: Brazil, Peru,the Philippines, and the United States. Arch Sex Behav, 1998 Feb;27(1):31-56

4. Bailey JM, Nothnagel J, Wolfe M. Retrospectively measured individual differences in childhood sex-typed behavior among gay men: correspondence between self- and maternal reports. Arch Sex Behav, 1995 Dec;24(6):613-22

5. Bailey JM, Miller JS, Willerman L. Maternally rated childhood gender nonconformity in homosexuals and heterosexuals. Arch Sex Behav, 1993 Oct;22(5):461-9

6. Phillips G, Over R. Differences between heterosexual, bisexual, and lesbian women in recalled childhood experiences. Arch Sex Behav, 1995 Feb;24(1):1-20

7. Lehmann JB, Lehmann CU, Kelly PJ. Development and health care needs of lesbians. J Womens Health 1998 Apr;7(3):379-87

8. Isay RA. Gender in homosexual boys: some developmental and clinical considerations. Psychiatry, 1999 Summer;62(2):187-94

9. Rekers GA, Mead SL, Rosen AC, Brigham SL. Family correlates of male childhood gender disturbance. J Genet Psychol, 1983 Mar;142(1st Half):31-42

10. Rekers GA, Swihart JJ. The association of gender identity disorder with parental separation. Psychol Rep, 1989 Dec;65(3 Pt 2):1272-4

11. Zucker KJ, Lightbody S, Pecore K, Bradley SJ, Blanchard R. Birth order in girls with gender identity disorder. Eur Child Adolesc Psychiatry, 1998 Mar;7(1):30-5

12. Cameron P, Cameron K. Children of homosexual parents report childhood difficulties. Psychol Rep, 2002 Feb;90(1):71-82

13. Dawood K, Pillard RC, Horvath C, Revelle W, Bailey JM. Familial aspects of male homosexuality. Arch Sex Behav, 2000 Apr;29(2):155-63

14. Cameron P, Cameron K. Does incest cause homosexuality? Psychol Rep, 1995 Apr;76(2):611-21

15. Phillips SH. The overstimulation of everyday life: I. New aspects of male homosexuality. J Am Psychoanal Assoc, 2001 Fall;49(4):1235-67

16. Blumenthal E. We all need our tails to lean on. An analysis of a latency-age girl with a gender identity disorder. Psychoanal Study Child, 1998;53:181-98

17. Gilmore K. Gender identity disorder in a girl: insights from adoption. J Am Psychoanal Assoc, 1995;43(1):39-59 
18. Loeb LR. Analysis of the transference neurosis in a child with transsexual symptoms J Am Psychoanal Assoc, 1992;40(2):587-605

19. Loeb L, Shane M. The resolution of a transsexual wish in a five-year-old boy. J Am Psychoanal Assoc, 1982;30(2):419-34

20. Meyer JK. The theory of gender identity disorders. J.Am Psychoanal Assoc, 1982;30(2):381-418

21. McDevitt JB. A childhood gender identity disorder. Analysis, preoedipal determinants, and therapy in adolescence. Psychoanal Study Child, 1995;50:79-105

22. Chused JF. Male gender identity and sexual behaviour. Int J Psychoanal, 1999 Dec;80 ( Pt 6):1105-17

23. Haber $\mathrm{CH}$. The psychoanalytic treatment of a preschool boy with a gender identity disorder. J Am Psychoanal Assoc, 1991;39(1):107-29

24. Bailey JM, Dunne MP, Martin NG. Genetic and environmental influences on sexual orientation and its correlates in an Australian twin sample. J Pers Soc Psychol, 2000 Mar;78(3):524-36

25. Paterson AD. X-chromosome workshop. Psychiatr Genet 1998 Summer;8(2):121-6

26. Zucker KJ,Beaulieu N,Bradley SJ,Grimshaw GM, Wilcox A. Handedness in boys with gender identity disorder. J Child Psychol Psychiatry, 2001 Sep;42(6):767-76

27. Zucker KJ, Wild J, Bradley SJ, Lowry CB. Physical attractiveness of boys with gender identity disorder. Arch Sex Behav, 1993 Feb;22(1):23-36

28. Roper WG. The etiology of male homosexuality. Med Hypotheses, 1996 Feb;46(2):85-8

29. Ajuriaguerra J. Manual de Psiquiatría infantil, Toray-Masson. Barcelona.1980.

30. Lebovici S. Diatkine R., Soulé M. Tratado de psiquiatría del niño y del adolescente. Tomo II. Biblioteca Nueva. Madrid 1988.

31. Stoller R.J. cit. en Fernández Rivas y González Torres «Objeto transicional y fetiche en el trastorno de identidad sexual en el niño» Rev. Psiq. Infanto-Juvenil. nº1 2001 pp.16-21.

32. Lacan J. La Familia. Homo Sapiens. Buenos Aires.1.977.

33. Fernández Rivas A., González Torres «Objeto transicional y fetiche en el trastorno de identidad sexual en el niño». Rev.de Psiquiatría Infanto-juvenil; $\mathrm{n}^{\circ} 1 / 2001$; enmar;pp.16-21.

* Psiquiatra Jefe de Servicio. Instituto Pere Mata.

Unidad Psiquiatría Universidad Rovira i Virgili.

Coordinador CSMIJ Tarragona. 